\title{
Quantized Consensus over Expander Networks and Communication Energy Minimization
}

\author{
Tao Li, Minyue Fu, Lihua Xie, and Ji-Feng Zhang
}

\begin{abstract}
Expander networks are highly connected sparse graphs, which play an important role in designing efficient communication networks. In this paper, we consider consensus control of discrete-time first-order agents with the communication graph being an expander network. Each agent has a real-valued state but can only exchange symbolic data with its neighbors. A distributed protocol is designed based on dynamic encoding and decoding with finite level uniform quantizers. The choice of the control parameters only depends on the number of agents, the maximum degree and the isoperimetric constant of the network. It is shown that under the protocol designed, averageconsensus can be achieved with an exponential convergence rate based on a single-bit information exchange between each pair of adjacent nodes at each time step. A performance index is given to characterize the total communication energy cost to achieve average-consensus and it is shown that the minimization of the communication energy cost leads to a tradeoff between the convergence rate and the number of quantization levels.
\end{abstract}

\section{INTRODUCTION}

In recent years, consensus problem with quantized communication has attracted more and more attention in the control community. In [1]-[3], average-consensus algorithms were designed with each agent having an integer-valued state. These algorithms can drive each agent to some interger approximation of the average of the initial states. In [4][7], quantized average-consensus problems were studied with real-valued states. In [4]-[6], algorithms with uniform and logarithmic quantizers of infinite levels were proposed to ensure the boundness of the consensus error. Furthermore, an algorithm based on dynamic quantization was proposed in [7]. The number of quantization levels, however, will diverge as the number of agents increases. In [8], random dither was used to make the quantization error a "white" noise. Then the distributed stochastic approximation method ([9][11]) was applied to achieve approximate average-consensus. For distributed cooperative control of multi-agent systems

Author for correspondence: Tao Li. Email: litao@amss.ac.cn

This work was supported by Singapore Millennium Foundation, Agency for Science, Technology and Research under Grant SERC 0521010037 , NSFC of China under Grants 60828006, 60674038, NSFC-Guangdong Joint Foundation under grant U0735003 and the Chinese National Laboratory of Space Intelligent Control.

$\mathrm{T}$. Li is on leave from the Academy of Mathematics and Systems Science, Chinese Academy of Sciences, and now is with School of EEE, Nanyang Technological University. Email: litao@amss.ac.cn

M. Fu is with School of EE\&CS, University of Newcastle, Australia. Email: minyue. fu@newcastle.edu.au

L. Xie is with the School of Electrical and Electronic Engineering, Nanyang Technological University, Singapore 639798. Email: elhxie@ntu.edu.sg

J. F. Zhang is with the Academy of Mathematics and Systems Science, Chinese Academy of Sciences, Beijing 100190, China. Email: jifeiss.ac.cn under communication constraints, though various kinds of algorithms have been presented as mentioned above, some fundamental theoretic problems are still open. For example, to achieve consensus of the network, how many bits of information does each pair of adjacent agents need to exchange at each time step ?

In [12], the average-consensus control was considered for discrete-time first-order undirected networks with a finite communication date rate. The communication between agents is based on dynamic encoding and decoding with finite-level quantization. It was shown that if the network is connected, then for any given number of quantization levels, the control gain and the scaling function can be chosen properly such that average-consensus can be asymptotically achieved. In particular, the control parameters can be properly chosen such that average-consensus can be achieved by using a single-bit quantizer. However, in [12], the algebraic connectivity of the communication graph is required to be known a prior for choosing suitable control parameters, which may be difficult when the communication graph is of a large scale.

In this paper, we show that instead of the exact value of the algebraic connectivity, we only need to know a positive lower bound of the algebraic connectivity for designing the protocol proposed in [12], and for an important class of complex networks, called expander networks, the lower bound of the algebraic connectivity can be worked out by using the isoperimetric constant. We also give a performance index to characterize the total communication energy cost to achieve average-consensus and show that the minimization of the communication energy cost leads to a tradeoff between the convergence rate and the number of quantization levels.

The remainder of this paper is organized as follows. In Section II, we present the network model and our proposed distributed protocol. In Section III, we recall the main results of [12]. In Section IV, we consider how to select the control parameters for an important class of complex networks called expander networks. In Section V, we give a performance index to characterize the total communication cost to achieve average-consensus and minimize its upper bound with respect to the control parameters. In Section VI, we give some concluding remarks.

The following notation will be used throughout this paper: 1 denotes a column vector with all ones. $I$ denotes the identity matrix with an appropriate size. For a given set $\mathcal{S}$, the number of its elements is denoted by $|\mathcal{S}|$. For a given vector or matrix $A$, its transpose is denoted by $A^{T}$, its Euclidean norm is denoted by $\|A\|_{2}$. For a given positive number $x$, the 
maximum integer less than or equal to $x$ is denoted by $\lfloor x\rfloor$; the minimum integer greater than or equal to $x$ is denoted by $\lceil x\rceil$.

\section{PROBlEM FORMULATION}

\section{A. Average-consensus problem}

In this paper, the dynamics of each agent is modeled as a discrete-time first-order integrator:

$x_{i}(t+1)=x_{i}(t)+h u_{i}(t), t=0,1, \ldots, i=1,2, \ldots, N$,

where $x_{i}(t) \in \mathbb{R}$ is the $i$ th agent's state, $u_{i}(t) \in \mathbb{R}$ is the $i$ th agent's control input, and $h$ is the control gain. The information flow among agents are modeled as an undirected graph $\mathcal{G}=\{\mathcal{V}, \mathcal{E}, \mathcal{A}\}$, where $\mathcal{V}=\{1,2, \ldots, N\}$ is the set of nodes with $i$ representing the $i$ th agent, $\mathcal{E}_{\mathcal{G}}$ is the set of edges and $\mathcal{A}=\left[a_{i j}\right] \in \mathbb{R}^{N \times N}$ is the weighted adjacency matrix of $\mathcal{G}$. An edge denoted by the unordered pair $(j, i)$ represents a communication channel from $j$ to $i$. Note that $\mathcal{A}$ is a symmetric matrix. For any $i, j \in \mathcal{V}, a_{i j}=a_{j i} \geq 0$, and $a_{i j}>0$ if and only if $j \in N_{i}$. Also, $\operatorname{deg}_{i}=\sum_{j=1}^{N} a_{i j}$ is called the degree of $i$, and $d^{*}=\max _{i} \operatorname{deg}_{i}$ is called the degree of $\mathcal{G}$. The Laplacian matrix of $\mathcal{G}$ is defined as $\mathcal{L}=$ $\mathcal{D}-\mathcal{A}$, where $\mathcal{D}=\operatorname{diag}\left(\operatorname{deg}_{1}, \ldots, \operatorname{deg}_{N}\right)$. A sequence of edges $\left(i_{1}, i_{2}\right),\left(i_{2}, i_{3}\right), \ldots,\left(i_{k-1}, i_{k}\right)$ is called a path from node $i_{1}$ to node $i_{k}$. The graph $\mathcal{G}$ is called a connected graph if for any $i, j \in \mathcal{V}$, there is a path from $i$ to $j$.

The dynamic system (1) together with the communication graph $\mathcal{G}$ is usually called a dynamic network ([13]). A group of controls $\mathcal{U}=\left\{u_{i}, i=1,2 \ldots, N\right\}$ is called a distributed protocol if for all $i, u_{i}(t)$ only depends on $x_{i}(s)$ and $x_{j}(s)$, $j \in N_{i}, s \leq t$. The average-consensus control is to design a distributed protocol for the dynamic network, such that for any initial values $x_{1}(0), \ldots, x_{N}(0)$, all the agents asymptotically reach an agreement with $\frac{1}{N} \sum_{j=1}^{N} x_{j}(0)$ when $t \rightarrow \infty$. That is, $\frac{1}{N} \sum_{j=1}^{N} x_{j}(0)$ can be computed asymptotically in a distributed manner.

\section{B. Protocol design}

In [12], we proposed a distributed protocol based on dynamic encoding and decoding.

The encoder $\Phi_{j}$ of the $j$ th agent is given by

$$
\left\{\begin{aligned}
\xi_{j}(0)= & 0 \\
\xi_{j}(t)= & g(t-1) \Delta_{j}(t)+\xi_{j}(t-1) \\
\Delta_{j}(t)= & q\left(g^{-1}(t-1)\left(x_{j}(t)-\xi_{j}(t-1)\right)\right) \\
& t=1,2, \ldots
\end{aligned}\right.
$$

where $\xi_{j}(t)$ is the internal state of $\Phi_{j}$, and $\Delta_{j}(t)$, which is the output of $\Phi_{j}$, is sent to the neighbors of the $j$ th agent. Here, $q(\cdot)$ is a finite-level uniform quantizer, and $g(t)>0$ is a scaling function.

The quantizer $q(\cdot): \mathbb{R} \rightarrow \Gamma$ is a map from $\mathbb{R}$ to the set $\Gamma$ of quantized levels. In this paper, we consider a finite-level uniform quantizer with

$$
\Gamma=\{0, \pm i, i=1,2, \ldots K\}
$$

The number of quantization levels is $2 K+1$. The associated quantizer $q(\cdot)$ is given by

$$
q(y)= \begin{cases}0, & -1 / 2<y<1 / 2 \\ i, & \frac{2 i-1}{2} \leq y<\frac{2 i+1}{2} \\ & i=1,2, \ldots, K-1 \\ K, & y \geq \frac{2 K-1}{2} \\ -q(-y), & y \leq-1 / 2\end{cases}
$$

Remark 1: The encoder $\Phi_{j}$ is a scaled difference encoder, and $\xi_{j}(t)$ is a one-step predictor. In this difference encoding algorithm, at each time step the "prediction error", $x_{j}(t)-$ $\xi_{j}(t-1)$, is quantized. Generally speaking, the amplitude of the prediction error is smaller than that of state $x_{j}(t)$ itself, so it can be represented by fewer bits.

Remark 2: If consensus is achieved, then the prediction error $x_{j}(t)-\xi_{j}(t-1)$ vanishes as $t \rightarrow \infty$. Therefore, intuitively the scaling function $g(t)$ should satisfy the following properties. On one hand, $g(t)$ should converge to zero asymptotically to make the quantizer persistently excited, such that the agents receive the their neighbors' information continuously. On the other hand, $g(t)$ should be large enough such that the quantizer will not be saturated.

For each communication channel $(j, i) \in \mathcal{E}$, the $i$ th agent receives $\Delta_{j}(t)$, and then uses the following decoder $\Psi_{j i}$ to estimate $x_{j}(t)$ :

$$
\left\{\begin{aligned}
\widehat{x}_{j i}(0)= & 0 \\
\widehat{x}_{j i}(t)= & g(t-1) \Delta_{j}(t)+\widehat{x}_{j i}(t-1), \\
& t=1,2, \ldots
\end{aligned}\right.
$$

where $\widehat{x}_{j i}(t)$ is the output of $\Psi_{j i}$.

Remark 3: When the output $\Delta_{j}(t)$ of the quantizer is zero, the $j$ th agent does not send any information, so for a $(2 K+$ 1)-level quantizer $q(\cdot)$, the communication channel $(j, i), i \in$ $N_{j}$ is required to be capable of transmitting $\left\lceil\log _{2}(2 K)\right\rceil$ bits without error at each time step. In particular, the quantizer $q(\cdot)$ given by

$$
q(y)= \begin{cases}0, & -1 / 2<y<1 / 2 \\ 1, & y \geq 1 / 2 \\ -1, & y \leq 1 / 2\end{cases}
$$

is a one-bit quantizer.

The distributed protocol is given by

$$
u_{i}(t)=\sum_{j \in N_{i}} a_{i j}\left(\widehat{x}_{j i}(t)-\xi_{i}(t)\right), t=0,1, \ldots,
$$

Denote

$$
\begin{aligned}
X(t) & =\left[x_{1}(t), \ldots, x_{N}(t)\right]^{T} \\
\widehat{X}(t) & =\left[\xi_{1}(t), \ldots, \xi_{N}(t)\right]^{T} \\
e(t) & =X(t)-\widehat{X}(t) \\
\delta(t) & =X(t)-J_{N} X(t),
\end{aligned}
$$

where $J_{N}=\frac{1}{N} \mathbf{1 1}^{T}$. 
Substituting the protocol (2), (4) and (6) into the system (1) leads to

$$
\left\{\begin{aligned}
X(t+1)= & (I-h \mathcal{L}) X(t)+h \mathcal{L} e(t), \\
\widehat{X}(t+1)= & g(t) Q\left[g^{-1}(t)(X(t+1)-\widehat{X}(t))\right] \\
& +\widehat{X}(t) .
\end{aligned}\right.
$$

\section{FINITE-LEVEL QUANTIZED CONSENSUS}

For the protocol designed and the resulting closed-loop system (8), it is shown that if the network is connected, then for any given number of quantization levels, the control gain and the scaling function can be chosen properly such that average-consensus can be asymptotically achieved with an exponential rate ([12]).

We need the following assumptions:

A1) $\mathcal{G}$ is connected.

A2) $\max _{i}\left|x_{i}(0)\right| \leq C_{x}, \max _{i}\left|\delta_{i}(0)\right| \leq C_{\delta}$, where $C_{x}$ and $C_{\delta}$ are known nonnegative constants.

Lemma 3.1: If Assumption A1) holds and $h<\frac{2}{\lambda_{N}(\mathcal{L})}$, then $\rho_{h}<1$, where $\rho_{h}=\max _{2 \leq i \leq N}\left|1-h \lambda_{i}(\mathcal{L})\right|$. Furthermore, if $h<\frac{2}{\lambda_{2}(\mathcal{L})+\lambda_{N}(\mathcal{L})}$, then $\rho_{h}=1-h \lambda_{2}(\mathcal{L})$.

Lemma 3.2: Suppose Assumptions A1)-A2) hold. For any given $h \in\left(0, \frac{2}{\lambda_{N}(\mathcal{L})}\right)$ and $\gamma \in\left(\rho_{h}, 1\right)$, let

$$
\begin{aligned}
K_{1}(h, \gamma) & =\left\lfloor M_{1}(h, \gamma)-\frac{1}{2}\right\rfloor+1 \\
M_{1}(h, \gamma) & =\frac{\sqrt{N} h^{2} \lambda_{N}^{2}(\mathcal{L})}{2 \gamma\left(\gamma-\rho_{h}\right)}+\frac{1+h \lambda_{N}(\mathcal{L})}{2 \gamma},
\end{aligned}
$$

and

$$
g_{0}>\max \left\{\frac{C_{x}}{K+\frac{1}{2}}, \frac{2\left(\gamma-\rho_{h}\right)\left(C_{\delta} \gamma+h C_{x} \lambda_{N}(\mathcal{L})\right)}{h \lambda_{N}(\mathcal{L})}\right\} .
$$

Then for any given $K \geq K_{1}(h, \gamma)$, under the protocol (2), (4) and (6) with the $(2 K+1)$-level uniform quantizer (3) and the scaling function $g(t)=g_{0} \gamma^{t}$, the closed-loop system (8) satisfies

$$
\lim _{t \rightarrow \infty} x_{i}(t)=\frac{1}{N} \sum_{j=1}^{N} x_{j}(0), i=1,2 \ldots, N .
$$

Theorem 3.1: Suppose Assumptions A1)-A2) hold. For any given $K \geq 1$, let

$$
\begin{array}{r}
\Omega_{K}=\left\{(\alpha, \beta) \mid \alpha \in\left(0, \frac{2}{\lambda_{2}(\mathcal{L})+\lambda_{N}(\mathcal{L})}\right), \beta \in\left(\rho_{\alpha}, 1\right),\right. \\
\left.M_{1}(\alpha, \beta)<K+\frac{1}{2}\right\},
\end{array}
$$

where $\rho_{\alpha}=\max _{2 \leq i \leq N}\left|1-\alpha \lambda_{i}(\mathcal{L})\right|$ and $M_{1}(\alpha, \beta)$ is defined by (10). Then, (i) $\Omega_{K}$ is nonempty. (ii) For any $(h, \gamma) \in \Omega_{K}$, under the protocol (2), (4) and (6) with $g(t)=g_{0} \gamma^{t}$ and the $(2 K+1)$-level uniform quantizer (3), the closed-loop system (8) satisfies

$$
\lim _{t \rightarrow \infty} x_{i}(t)=\frac{1}{N} \sum_{j=1}^{N} x_{j}(0), i=1,2 \ldots, N,
$$

where $g_{0}$ is a constant satisfying (11).
The convergence rate of average-consensus ([14]) is defined as

$$
r_{\text {asym }}=\sup _{X(0) \neq J_{N} X(0)} \lim _{t \rightarrow \infty}\left(\frac{\left\|X(t)-J_{N} X(0)\right\|_{2}}{\left\|X(0)-J_{N} X(0)\right\|_{2}}\right)^{1 / t} .
$$

For the convergence rate of our algorithm, we have the following theorem.

Theorem 3.2: Suppose the conditions of Lemma 3.2 hold, then under the protocol (2), (4) and (6) with the $(2 K+1)$-level uniform quantizer (3), the closed-loop system (8) satisfies

$$
\|\delta(t)\|_{2}=O\left(\gamma^{t}\right), t \rightarrow \infty,
$$

and $r_{\text {asym }} \leq \gamma$, where $\delta(t)$ is the consensus error defined by (7).

Remark 4: Theorem 3.2 gives an estimate for the convergence rate of the consensus. The smaller the $\gamma$, the faster the consensus error converges to zero. Note that $\gamma$ can be made arbitrarily close to $\rho_{h}$, which is the convergence rate for the case with perfect communication ([14]).

\section{PARAMETER SELECTION FOR EXPANDER NETWORKS}

From Theorem 3.1, it can be seen that to ensure averageconsensus by using any given $\left\lceil\log _{2}(2 K)\right\rceil$-bit quantizer, the spectrum radius and the algebraic connectivity have to be known a prior for the choice of the control gain $h$ and the scaling factor $\gamma$. In fact, we can show that by making the set $\Omega_{K}$ smaller, the choice of $h$ and $\gamma$ only depends on a positive lower bound of the algebraic connectivity and the degree of the communication graph. We have the following result.

Theorem 4.1: Suppose Assumptions A1)-A2) hold. For any given $K \geq 1$, let

$$
\begin{array}{r}
\bar{\Omega}_{K}=\left\{(h, \gamma) \mid h \in\left(0, \frac{1}{2 d^{*}}\right), \gamma \in\left(1-h \lambda_{*}, 1\right),\right. \\
\left.\bar{M}_{1}(h, \gamma)<K+\frac{1}{2}\right\}
\end{array}
$$

where

$$
\bar{M}_{1}(h, \gamma)=\frac{2 \sqrt{N} h^{2}\left(d^{*}\right)^{2}}{\gamma\left[\gamma-\left(1-h \lambda_{*}\right)\right]}+\frac{1+2 h d^{*}}{2 \gamma},
$$

and $\lambda_{*}$ is a positive lower bound of $\lambda_{2}(\mathcal{L})$. Then, $\bar{\Omega}_{K} \subseteq \Omega_{K}$ and $\bar{\Omega}_{K}$ is nonempty.

The proof of Theorem 4.1 is omitted here.

Below we show that for an important class of regular networks, a lower bound of the algebraic connectivity can be worked out by using other physical parameters.

Definition 4.1: ([15]) The isoperimetric constant or expander constant of an equally weighted graph $\mathcal{G}=\{\mathcal{V}, \mathcal{E}, \mathcal{A}\}$ on $N$ vertices is

$$
i_{c}(\mathcal{G})=\min \left\{\frac{|\partial \mathcal{F}|}{|\mathcal{F}|}: \mathcal{F} \subseteq \mathcal{V}, 0<|\mathcal{F}| \leq \frac{N}{2}\right\},
$$

where the boundary $\partial \mathcal{F}$ of $\mathcal{F}$ is the set of edges with one vertex in $\mathcal{F}$ and the other in $\mathcal{V}-\mathcal{F}$. 
Definition 4.2: ([15]) Let $c$ be a positive constant. A $d$-regular graph $\mathcal{G}=\{\mathcal{V}, \mathcal{E}, \mathcal{A}\}$ is called a $c$-expander, if $i_{c}(\mathcal{G}) \geq c$.

Expander graphs are highly connected sparse graphs, which play an important role in designing efficient communication networks ([16],[17]). From Corollary 2.3 of [18], a key property of a $c$-expander is

$$
\lambda_{2}(\mathcal{L}) \geq d-\sqrt{d^{2}-c^{2}} .
$$

For expander networks, we have the following result.

Theorem 4.2: Let $\mathcal{G}=\{\mathcal{V}, \mathcal{E}, \mathcal{A}\}$ be a $d$-regular $c$ expander. Suppose Assumption A2) holds. For any given $h \in\left(0,(2 d)^{-1}\right)$ and $\gamma \in\left(1-h\left(d-\sqrt{d^{2}-c^{2}}\right), 1\right)$, let

$$
\begin{aligned}
K_{2}(h, \gamma)= & \left\lfloor M_{2}(h, \gamma)-\frac{1}{2}\right\rfloor+1, \\
M_{2}(h, \gamma)= & \frac{2 \sqrt{N} h^{2} d^{2}}{\gamma\left[\gamma-1+h\left(d-\sqrt{d^{2}-c^{2}}\right)\right]} \\
& +\frac{1+2 h d}{2 \gamma},
\end{aligned}
$$

and

$$
g_{0}>\max \left\{\frac{C_{x}}{K+\frac{1}{2}}, \frac{4 h d\left(d^{2}+\sqrt{d^{2}-c^{2}}\right)\left(C_{\delta}+C_{x}\right)}{h c^{2}}\right\} .
$$

Then for any given $K \geq K_{2}(h, \gamma)$, under the protocol (2), (4) and (6) with the $(2 K+1)$-level uniform quantizer (3) and the scaling function $g(t)=g_{0} \gamma^{t}$, the closed-loop system (8) satisfies

$$
\lim _{t \rightarrow \infty} x_{i}(t)=\frac{1}{N} \sum_{j=1}^{N} x_{j}(0), i=1,2 \ldots, N .
$$

Proof: Note that for a $c$-expander, Assumption A1) holds. From the property of graph Laplacian ([19]), we know that $\lambda_{N}(\mathcal{L}) \leq 2 d$. Then by Lemma 3.1 and $h \in\left(0,(2 d)^{-1}\right)$, we get

$$
\rho_{h}=1-h \lambda_{2}(\mathcal{L}) .
$$

This together with (13), (14), (15), (9) and (10) leads to

$$
K_{2}(h, \gamma) \geq K_{1}(h, \gamma) \text {. }
$$

By (18) and the property of graph Laplacian ([19]), we have

$$
\gamma-\rho_{h, \mathcal{G}} \leq 1-\left(1-h \lambda_{2}(\mathcal{L})\right) \leq 2 h d,
$$

which together with $h \lambda_{N}(\mathcal{L})<1$, and $\lambda_{N}(\mathcal{L}) \geq \lambda_{2}(\mathcal{L}) \geq$ $d-\sqrt{d^{2}-c^{2}}$ gives

$$
\begin{aligned}
& \frac{2\left(\gamma-\rho_{h, \mathcal{G}}\right)\left(C_{\delta} \gamma+h C_{x} \lambda_{N}(\mathcal{L})\right)}{h \lambda_{N}(\mathcal{L})} \\
\leq & \frac{4 h d\left(d+\sqrt{d^{2}-c^{2}}\right)\left(C_{\delta}+C_{x}\right)}{h c^{2}} .
\end{aligned}
$$

By this, (16), (11), (19) and Lemma 3.2, we have (17).

Theorem 4.3: Let $\mathcal{G}=\{\mathcal{V}, \mathcal{E}, \mathcal{A}\}$ be a $d$-regular $c$ expander. Suppose Assumption A2) holds. For any given $K \geq 1$, let $\Omega_{K}^{c}=\left\{(\alpha, \beta) \mid \alpha \in\left(0, \frac{1}{2 d}\right), \beta \in(1-\alpha(d-\right.$ $\left.\left.\left.\sqrt{d^{2}-c^{2}}\right), 1\right), M_{2}(\alpha, \beta)<K+\frac{1}{2}\right\}$, where $M_{2}(\alpha, \beta)$ is given by (15). Then, (i) $\Omega_{K}^{c}$ is nonempty. (ii) For any given $(h, \gamma) \in \Omega_{K}^{c}$, under the protocol (2), (4) and (6) with $g(t)=g_{0} \gamma^{t}$ and the $(2 K+1)$-level uniform quantizer (3), the closed-loop system (8) satisfies

$$
\lim _{t \rightarrow \infty} x_{i}(t)=\frac{1}{N} \sum_{j=1}^{N} x_{j}(0), i=1,2 \ldots, N,
$$

where $g_{0}$ is a constant satisfying (16).

Proof: (i) Noting that

$$
\lim _{\alpha \rightarrow 0}\left[\frac{2 \sqrt{N} \alpha d^{2}}{d-\sqrt{d^{2}-c^{2}}}+\frac{1+2 \alpha d}{2}\right]=\frac{1}{2},
$$

we know that for any given $K \geq 1$, there exists $\alpha^{*} \in\left(0, \frac{1}{2 d}\right)$ such that

$$
\frac{2 \sqrt{N} \alpha^{*} d^{2}}{d-\sqrt{d^{2}-c^{2}}}+\frac{1+2 \alpha^{*} d}{2}<K+\frac{1}{2} .
$$

By this and (15), we get

$$
\lim _{\gamma \rightarrow 1} M_{2}\left(\alpha^{*}, \gamma\right)=\frac{2 \sqrt{N} \alpha^{*} d^{2}}{d-\sqrt{d^{2}-c^{2}}}+\frac{1+2 \alpha^{*} d}{2} .
$$

Then by (20), we know that there exists $\gamma^{*} \in\left(1-\alpha^{*}(d-\right.$ $\left.\sqrt{d^{2}-c^{2}}\right), 1$ ), such that

$$
M_{2}\left(\alpha^{*}, \gamma^{*}\right)<K+\frac{1}{2}
$$

Therefore $\left(\alpha^{*}, \gamma^{*}\right) \in \Omega_{K}^{c}$, that is, $\Omega_{K}^{c}$ is nonempty.

(ii) For any $(h, \gamma) \in \Omega_{K}^{c}$, by the definition of $\Omega_{K}^{c}$, we know that

$$
\frac{1}{2}<M_{2}(h, \gamma)<K+\frac{1}{2}
$$

Thus, by (14), one gets $K_{2}(h, \gamma) \leq K$, which together with Theorem 4.2 leads to the conclusion of the theorem.

To choose $(h, \gamma)$ from $\Omega_{K}^{c}$ for any given $K \geq 1$, we have the following algorithm.

\section{Algorithm:}

(i) Choose a constant $\epsilon_{0} \in(0,1)$.

(ii) Choose the control gain $h \in\left(0, \widetilde{h}_{c}\left(\epsilon_{0}\right)\right)$, where

$$
\begin{aligned}
\widetilde{h}_{c}\left(\epsilon_{0}\right)= & \min \left\{\frac{1}{2 d}, 2 K \epsilon_{0}\left(\frac{\sqrt{N} d^{2}\left(d+\sqrt{d^{2}-c^{2}}\right)}{c^{2}}\right.\right. \\
& \left.\left.+2 d \epsilon_{0}+2(2 K+1) d \epsilon_{0}\left(1-\epsilon_{0}\right)\right)^{-1}\right\}
\end{aligned}
$$

(iii) Let $\gamma=1-\left(1-\epsilon_{0}\right) h\left(d+\sqrt{d^{2}-c^{2}}\right)$.

Remark 5: A Ramanujan graph $\mathcal{G}$ is defined as a $d$-regular graph with $\lambda_{2}(\mathcal{L}) \geq d-2 \sqrt{d-1}$ ([20]-[21]). Ramanujan graphs are expanders which achieve the largest possible algebraic connectivity, and so are optimal expanders from the spectral point of view ([15], [22]). Recently, it is found that in fact, Ramanujan graphs are close to optimal graphs with best synchronizability ([16]). For a Ramanujan graph $\mathcal{G}$ with $d \geq 3, \lambda_{2}(\mathcal{L}) \geq 3-2 \sqrt{2}$. This property is of particular importance, since $\lambda_{2}(\mathcal{L})$ is lower bounded, independent of the graph $\mathcal{G}$. So by Theorem 4.3, for the case with a $d$-regular Ramanujan graphs with $d \geq 3$, we can choose $h$ and $\gamma$, such that $h \in\left(0, h_{c}\left(\epsilon_{0}\right)\right)$ and $\gamma=1-\left(1-\epsilon_{0}\right)(3-2 \sqrt{2}) h$, where

$$
\widetilde{h}_{c}\left(\epsilon_{0}\right)=\min \left\{\frac{1}{2 d},\right.
$$




$$
\left.\frac{2 K \epsilon_{0}}{\frac{\sqrt{N} d^{2}}{3-2 \sqrt{2}}+2 d \epsilon_{0}+2(2 K+1) d \epsilon_{0}\left(1-\epsilon_{0}\right)}\right\},
$$

for any given $K \geq 1$.

\section{Minimization OF COMMUNicATION ENERGy COST}

Average-consensus protocols can be viewed as distributed least-mean-squares estimation algorithms in sensor networks ([23]). Limited power is perhaps the most critical constraint for applications of wireless sensor networks. Simulations show that for a large-scale sensor network, communications between nodes consume far more power than computation ([24]). For power saving, reducing the communication load for distributed estimation is a critical issue. This motivates us to consider how to minimize the total communication energy cost for average-consensus. We define the convergence time constant as $\tau_{\text {asym }}=\left(\ln \left(1 / r_{\text {aysm }}\right)\right)^{-1}$, which gives the asymptotic number of steps for the consensus error to decrease by $1 / e .{ }^{1}$ For a $(2 K+1)$-level quantizer, at each time instant, $\left\lceil\log _{2}(2 K)\right\rceil$ bits are sent by each sending node and are received by each receiving node. For simplicity, we assume that the transmission power and receiving energy cost for each node are the same. Therefore, if a $\left(2 K_{1}(h, \gamma)+1\right)$ level quantizer is used, then the communication energy of the whole sensor networks to achieve consensus is given by

$$
\Phi=B_{h}\left(c_{1}|\mathcal{V}|+2 c_{2}\left|\mathcal{E}_{\mathcal{G}}\right|\right)
$$

where $B_{h}=\left\lceil\log _{2}\left(2 K_{1}(h, \gamma)\right)\right\rceil \tau_{\text {asym }}$, and $c_{1}$ and $c_{2}$ are respectively the energy costs for transmitting and receiving a single bit data. Minimizing $\Phi$ is equivalent to minimizing $B_{h}$. By Theorem 3.2, we know that

$$
B_{h} \leq B_{h}^{*},
$$

where

$$
B_{\mathcal{G}, h}^{*}=\left\lceil\log _{2}\left(2 K_{1}(h, \gamma)\right)\right\rceil(\ln (1 / \gamma))^{-1},
$$

which is a function of $\gamma$ for given network topology $\mathcal{G}$ and the control gain $h$. The smaller the $\gamma$, the smaller the $(\ln (1 / \gamma))^{-1}$, but the larger the $\left\lceil\log _{2}\left(2 K_{1}(h, \gamma)\right)\right\rceil$. Choosing $\gamma$ to minimize $B_{h}^{*}$, we can get a good solution for $\gamma$.

Example 1: We consider a network with 30 nodes and $0-1$ weights ${ }^{2}$ shown in Fig. 1. The edges of the graph are randomly generated according to $P\left\{(i, j) \in \mathcal{E}_{\mathcal{G}}\right\}=\frac{1}{2}$, for any unordered pair $(i, j)$. Suppose $h$ is chosen as 0.2 . The curve of $B_{0.2}^{*}$ with respect to $\gamma$ is given by Fig. 2. It can be seen that $B_{0.2}^{*}$ arrives at the minimum when $\gamma$ is approximately 0.8896 . The curves of $B_{h}^{*}$ when $h=0.5$ and $h=0.8$ are shown in Fig. 3. The discontinuity of the curves is due to the rounding up and down operators. It is shown that the suboptimal value of $\gamma$ decreases as $h$ increases.

Remark 6: In many scenarios ([25]), the dynamics of a group of autonomous robots under feedback linearization can be modeled as continuous-time first-order integrators

$$
\dot{x}_{i}=u_{i}(t), t \geq 0, i=1,2, \ldots, N .
$$

\footnotetext{
${ }^{1}$ Note that the term convergence time is used in [14], but we believe that calling it the convergence time constant is more appropriate.

${ }^{2} 0-1$ weights means that $a_{i j}=1$, if $(i, j) \in \mathcal{E}$, otherwise, $a_{i j}=0$.
}

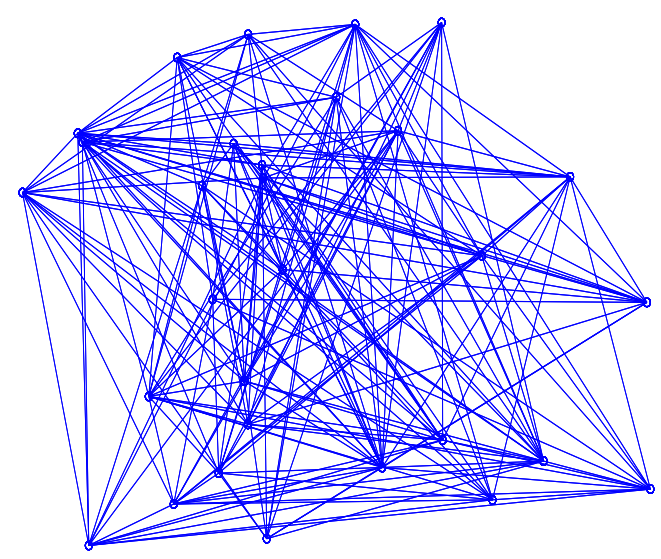

Fig. 1. Network topology of Example 1.

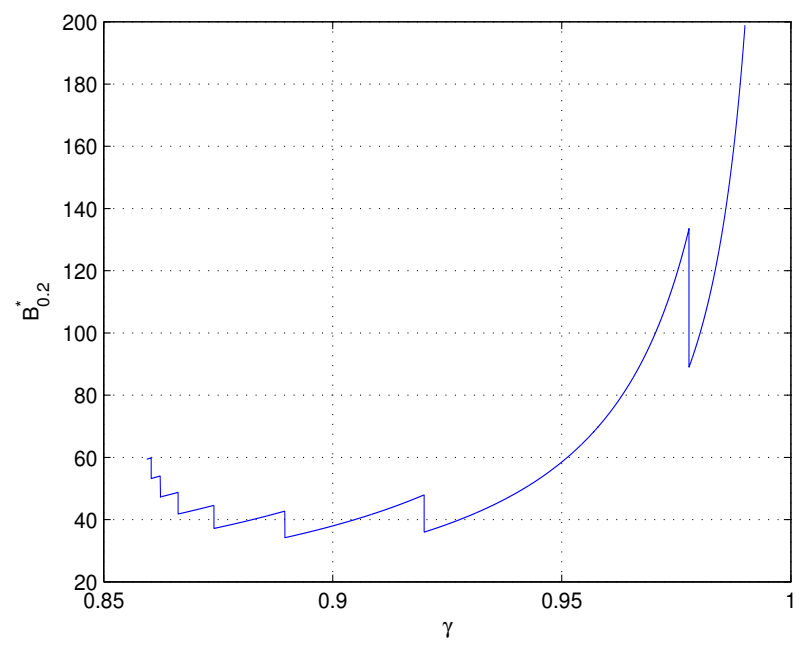

Fig. 2. Curves of $B_{0.2}^{*}$ versus $\gamma$.

Then the model (1) can be viewed as the associated discretetime model with zero-order holder functions, where $h$ is the sampling period. In the case of sampled-data control, besides the total communication load, we also want to minimize the bit rate requirement for each communication channel. From Lemma 3.2, we know that the bit rate of each communication channel can be made as low as

$$
\inf _{h \in\left(0,2\left(\lambda_{N}(\mathcal{L})\right)^{-1}\right), \gamma \in\left(\rho_{h, \mathcal{G}}, 1\right)} \frac{\left\lceil\log _{2}\left(2 K_{1}(h, \gamma)\right)\right\rceil}{h} .
$$

\section{CONCLUding REMARKS}

In this paper, the average-consensus control problem has been considered for an undirected network of discrete-time first-order agents under finite bit-rate communication. Based on scaled uniform quantization, a dynamic difference encoding and decoding scheme is used for the communication between each pair of agents. A distributed protocol has been proposed, where the control input of each agent is a weighted 


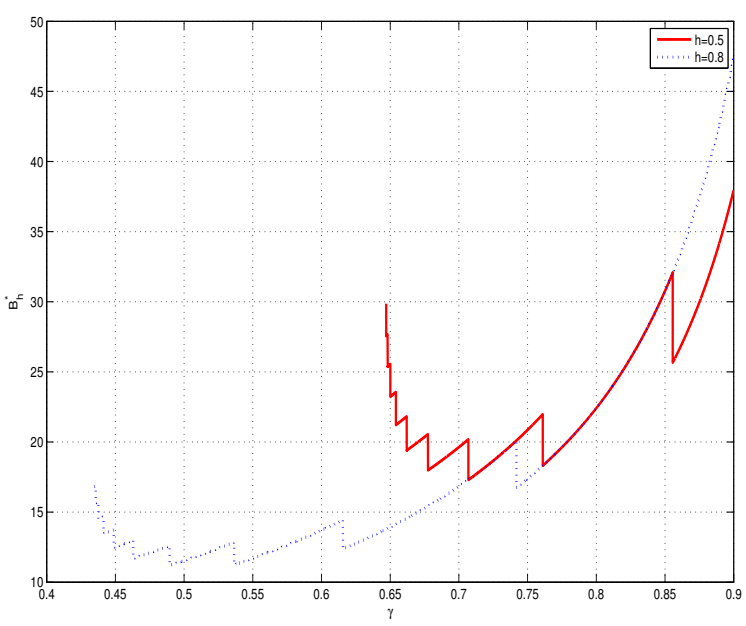

Fig. 3. Curves of $B_{h}^{*}$ versus $\gamma$ when $h=0.5$ and $h=0.8$.

sum of the difference between the estimate of its neighbor's state and the internal state of its own encoder. We show that when the communication graph belongs to the family of expander graphs, the choice of the control parameter only depends on the number of agents, the maximum degree and the isoperimetric constant of the network. It has been shown that under the protocol designed, average-consensus can be achieved with an exponential convergence rate based on a single-bit information exchange between each pair of adjacent nodes at each time step.

\section{REFERENCES}

[1] A. Kashyap, T. Basar and R. Srikant, "Consensus with quantized information updates", Proc. of the 45th IEEE Conference on Decision and Control, San Diego, USA, December 13-15, pp. 2728-2733, 2006.

[2] A. Kashyap, T. Basar and R. Srikant, "Quantized consensus", Automatica, Vol. 43, No. 7, pp. 1192-1203, 2007.

[3] A. Nedic, A. Olshevsky, A. Ozdaglar and J. N. Tsitsiklis, "On Distributed Averaging Algorithms and Quantization Effects", Arxiv preprint arXiv:0711.4179, 2007.

[4] R. Carli, F. Fagnani, P. Frasca, T. Taylor and S. Zampieri, "Average consensus on networks with transmission noise or quantization", Proc. of European Control Conference 2007, pp. 1852-1857, 2007.

[5] R. Carli, F. Fagnani, P. Frasca and S. Zampieri, "A probabilistic analysis of the average consensus algorithm with quantized communication", Proc. of the 17th IFAC World Congress, Seoul, Korea, July, 6-11, 2008.

[6] D. V. Dimarogonas and K. H. Johansson, "Quantized agreement under time-varying communicaiton topology", Proc. of American Control Conference 2008, Seattle, WA, June, 11-13, pp. 4376-4381, 2008.

[7] R. Carli, F. Fagnani, P. Frasca and S. Zampieri, "Efficient quantized techniques for consensus algorithms", NeCST workshop, Nancy, France, 2007.

[8] S. Kar and J. M. F. Moura, "Distributed consensus algorithms in sensor networks: quantized data", Arxiv preprint arXiv:0712.1609, 2007.

[9] M. Huang and J. H. Manton, "Coordination and consensus of networked agents with noisy measurement: stochastic algorithms and asymptotic behavior", SIAM Journal on Control and Optimization, Vol. 48, No. 1, Special issue on Control and Optimization in Cooperative Networks, pp. 134-161, 2009.

[10] M. Huang and J. H. Manton, "Stochastic approximation for consensus seeking: mean square and almost sure convergence", Proc. of 46th IEEE Conference on Decision and Control, New Orleans, LA, December 12-14, pp. 306-311, 2007.
[11] T. Li and J. F. Zhang, "Consensus conditions of multiagent systems with time-varying topologies and stochastic communicaiton noises", provisionally accepted by IEEE Transactions on Automatic Control, 2009. (See http://lsc.amss.ac.cn/ jif/paper/[J68].pdf)

[12] T. Li, M. Fu, L, Xie and J. F. Zhang,"Distributed consensus of multiagent systems with finite-level quantization", Proc. of the 7th Asian Control Conference, HongKong, August, 27-29, 2009.

[13] R. Olfati-Saber and R. M. Murray, "Consensus problem in networks of agents with switching topology and time-delays," IEEE Trans. on Automatic Control, Vol. 49, No. 9, pp. 1520-1533, 2004.

[14] L. Xiao and S. Boyd, "Fast linear iterations for distributed averaging", Systems and Control Letters, Vol. 53, No. 1, pp. 65-78, 2004.

[15] G.P. Davidoff, P. Sarnak and A. Valette, Elementary Number Theory Group Theory and Ramanujan Graphs, London: Cambridge University Press, 2003

[16] L. Donetti, P. I. Hurtado and M. A. Muñoz, "Entangled Networks, Synchronization, and Optimal Network Topology", Physical Review Letters, Vol. 95, No. 18, pp. 188701, 2005.

[17] F. Bien, "Construction of telephone networks by group representations", Notices of the Amer. Math. Society, Vol. 36, No. 1, pp. 5-22, 1989.

[18] A. Berman and X. D. Zhang, "Lower bounds for the eigenvalues of Laplacian matrices", Linear Algebra and its Applications,

[19] C. Godsil and G. Royle, Algebraic Graph Theory, New York: SpringerVerlag, 2001.

[20] A. Lubotzky, R. Phillips and P. Sarnak, "Ramanujan graphs", Combinatorica, Vol. 8, No. 3, pp. 261-277, 1988.

[21] M. R. Murty, "Ramanujan Graphs", Journal-Ramanujan Mathematical Society, Vol. 18, No. 1, pp. 33-52, 2003.

[22] R. Olfati-Saber, "Algebraic Connectivity Ratio of Ramanujan Graphs", 2007 American Control Conference, pp. 4619-4624, 2007. Vol. 316, No. 1, pp. 13-20, 2000.

[23] L. Xiao, S. Boyd and S. Lall, "A scheme for robust distributed sensor fusion based on average consensus", Proc. of the 4th International Symposium on Information Processing in Sensor Networks, pp. 63-70, 2005.

[24] V. Shnayder, M. Hempstead, B. Chen, G. W. Allen, M. Welsh, "Simulating the power consumption of large-scale sensor network applications", Proceedings of the 2nd International Conference on Embedded Networked Sensor Systems, pp. 188-200, 2004.

[25] W. Ren and R. W. Beard, Distributed Consensus in Multi-vehicle Cooperative Control, London: Springer-Verlag, 2008. 